\section{Artikel 43}

Nachdem die gesetzmäßige Gewalt tatsächlich in die Hände des Besetzenden übergegangen ist, hat dieser alle von ihm abhängenden Vorkehrungen zu treffen, um nach Möglichkeit die öffentliche Ordnung und das öffentliche Leben wiederherzustellen und aufrechtzuerhalten, und zwar, soweit kein zwingendes Hindernis besteht, unter Beachtung der Landesgesetze.

\section{Artikel 46}

Die Ehre und die Rechte der Familie, das Leben der Bürger und das Privateigentum sowie die religiösen Überzeugungen und gottesdienstlichen Handlungen sollen geachtet werden. Das Privateigentum darf nicht eingezogen werden.

\section{Artikel 47}

Die Plünderung ist ausdrücklich untersagt.

\section{Artikel 50}

Keine Strafe in Geld oder anderer Art darf über eine ganze Bevölkerung wegen der Handlungen einzelner verhängt werden, für welche die Bevölkerung nicht als mitverantwortlich angesehen werden kann.

\section{Artikel 56}

Das Eigentum der Gemeinden und der dem Gottesdienste, der Wohltätigkeit, dem Unterrichte, der Kunst und der Wissenschaft gewidmeten Anstalten, auch wenn diese dem Staate gehören, ist als Privateigentum zu behandeln. Jede Beschlagnahme, jede absichtliche Zerstörung oder Beschädigung von derartigen Anlagen, von geschichtlichen Denkmälern oder von Werken der Kunst und Wissenschaft ist untersagt und soll geahndet werden.

\title{
Wissenschaftliches Symposium: Die Europäische Union im 21. Jahrhundert
}

\author{
Bernhard Rinke*
}

Im März diesen Jahres konnte die Europäische Union ein mehr als nur beachtenswertes Jubiläum begehen: Den fünfzigsten Jahrestag der Unterzeichnung der sog. »Römischen Verträge « am 25. März 1957, mit der die bis dahin allein in der Europäischen Gemeinschaft für Kohle und Stahl vereinigten Staaten - Belgien, Frankreich, Italien, Luxemburg, die Niederlande und die Bundesrepublik - die Europäische Wirtschaftsgemeinschaft (EWG) und die Europäische Atomgemeinschaft (EAG) gründeten. Im historischen Rückblick markiert dieses Datum zweifellos einen grundlegenden Meilenstein des europäischen Integrationsprozesses, in dessen Verlauf sich durch Höhen und Tiefen die heutige Europäische Union als Staatenverbund mit weltweit einzigartiger Integrationsdichte entwickelt hat. Am Beginn dieses Prozesses stand - angesichts der Millionen von Toten, Verwundeten und Vertriebenen sowie der ungeheuren Zerstörungen und Gräueltaten des 1. und 2. Weltkriegs - in erster Linie der Wunsch, Krieg als Mittel der Politik ein für allemal zu überwinden. Als innereuropäische Friedensgemeinschaft hat sich die Europäische Gemeinschaft/Europäische Union in den zurückliegenden Jahrzehnten bewährt. Doch an der Schwelle zum 21. Jahrhundert geht es nicht mehr nur um Frieden und Sicherheit in der Europäischen Union, sondern - angesichts vielfältiger alter und neuer sicherheitspolitischer Herausforderungen und Bedrohungen - auch um Frieden und Sicherheit für, mit und durch die Europäische Union. ${ }^{1}$

\footnotetext{
* Dr. Bernhard Rinke, Institut für Friedensforschung und Sicherheitspolitik an der Universität Hamburg (IFSH).

1 Vgl. auch Ehrhart, Hans-Georg et al (Hrsg.): Die Europäische Union im 21. Jahrhundert. Theorie und Praxis europäischer Außen-, Sicherheits- und Friedenspolitik, Wiesbaden 2007.
}

Darin einig waren sich auch die Teilnehmer eines wissenschaftlichen Symposiums unter dem Titel »Die Europäische Union im 21. Jahrhundert«, dass aus Anlass des 60. Geburtstages von Prof. Dr. Dr. h.c. Reinhard Meyers am 27. April 2007 vom Institut für Politikwissenschaft der Westfälischen Wilhelms-Universität Münster veranstaltet wurde. Unter Moderation von Bernhard Rinke diskutierten dabei Dr. Hans-Georg Ehrhart (stellv. Leiter ZEUS), Prof. Dr. Dr. h.c. Wichard Woyke (Professor für Europapolitik, Universität Münster), Prof. Dr. Wilfried Loth (Professor für Neuere und Neueste Geschichte, Universität Duisburg-Essen) und Prof. Dr. Hajo Schmidt (Direktor des Instituts Frieden und Demokratie der FernUniversität Hagen) über die Perspektiven der Europäischen Union im 21. Jahrhundert und ihre friedenspolitische Rolle auf dem Weg zu einer mehr und mehr operativ ausgerichteten Außenund Sicherheitspolitik.

Wichard Woyke fokussierte sich in seinen Ausführungen auf die Zukunft der außen- und sicherheitspolitischen Abschnitte des Europäischen Verfassungsvertrags. Als voraussichtlichen Ausweg aus der gegenwärtigen Verfassungskrise skizzierte er die Umrisse eines »Minivertrags«, der angesichts der großen internationalen Nachfrage nach dem Akteur EU allerdings unbedingt zu einer Stärkung der außen- und sicherheitspolitischen Handlungsfähigkeit der EU führen müsse. Entsprechend plädierte er nachhaltig dafür, das im Verfassungsvertrag vorgesehene Amt eines Europäischen Außenministers zu schaffen. Unter Verweis auf die unterschiedlichen außenpolitischen Traditionen und sicherheitspolitischen Ausrichtungen der EU-Mitgliedstaaten skizzierte er allerdings zugleich das 
Szenario einer differenzierten Integration in der Außen- und Sicherheitspolitik, wie sie auch im Verfassungsvertrag mit den Instrumenten der »verstärkten« und »engeren Zusammenarbeit« einzelner EU-Mitgliedstaaten angelegt sei.

Auch Hans-Georg Ehrhart sah in seinem Diskussionsbeitrag »keine Alternative« zu einer stärkeren Rolle der Europäischen Union als Akteur im internationalen System. Vor allem gelte es, so Ehrhart weiter, bestehende »Implementationsdefizite « bei der Umsetzung der europäischen Außen- und Sicherheitspolitik zu beheben. Im gleichen Kontext verwarf er den Begriff der »Militarisierung Europas«. Zwar sei die EU kein ausschließlich auf zivile Mittel setzende Zivilmacht mehr. Allerdings verfolge sie ebenso wenig eine militärische Machtpolitik im Stile und mit den Mitteln einer klassischen Großmacht. Vielmehr handele es sich bei der EU um eine »Friedensmacht «, welche - unter Einbeziehung militärischer Zwangsmittel - »die ganze Palette ihrer Fähigkeiten für die Prävention und konstruktive Bearbeitung von Gewaltkonflikten « einbringe.

Dieser Einschätzung widersprach Hajo Schmidt, indem er auf Erkenntnisse zahlreicher friedens- und konfliktwissenschaftlicher Untersuchungen der letzten Zeit verwies. Demnach lasse insbesondere der Anspruch globaler Interventionsfähigkeit die EU-Sicherheits- und Friedenspolitik ambivalent erscheinen; eine »mehr als schleichende Militarisierung « lasse sich auch institutionell und finanziell, im Vergleich zu Aufwendungen für das zivile Konfliktmanagement, kaum bestreiten. So sei denn auch das im Verfassungsvertrag formulierte Selbstverständnis der EU, eine Friedensmacht in Übereinstimmung mit der UN-Charta zu verkörpern, »sachlich immer weniger gedeckt «. Zwar werde »Frieden« als oberstes Ziel der EU bestätigt. Doch werde diese »zentrale Selbstfestlegung « konzeptionell nicht weiter geklärt. Überdies werde der Friedens- weitgehend durch einen »verteidigungs- und militärzentrierten Sicherheitsbegriff « ersetzt, der seinerseits kaum Bezüge zu gewaltarmen, zivilen Konfliktmanagementformen erkennen lasse.

Mit Blick auf die Debatte über die künftige Gestaltung der Europäischen Union mahnte Wilfried Loth schließlich, dass sich die alte Begründung des europäischen Projekts - die Schaffung einer europäischen Friedensordnung - keineswegs »erledigt« habe, sondern weiterhin fundamentale Aufgabe bleibe. Zugleich wandte er sich gegen eine rückblickende »Glorifizierung « des Integrationsprozesses. So habe sich das Einigungswerk, ausgelöst durch das Scheitern ambitionierter Projekte wie der Europäischen Politischen Gemeinschaft (EPG) und der Europäischen Verteidigungsgemeinschaft (EVG), vor Abschluss der Römischen Verträge in einer sehr viel tieferen Krise als heute befunden. Als Kern der aktuellen Krise bezeichnete er derweil die fehlende Legitimität der EU. Daher sei es notwendig, den Dialog zwischen den europäischen Eliten und den Bürgern der Union über die politischen Zielsetzungen des europäischen Zusammenschlusses zu forcieren.

\title{
Friedensnobelpreis 2005
}

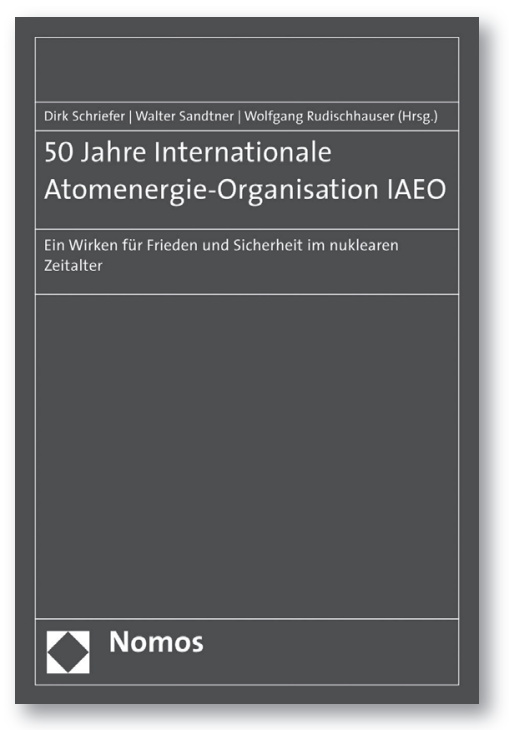

\author{
50 Jahre Internationale Atomenergie-Organisation IAEO \\ Ein Wirken für Frieden und Sicherheit im nuklearen Zeitalter \\ Herausgegeben von Dirk Schriefer, Walter Sandtner und \\ Wolfgang Rudischhauser \\ 2006, 218 S., brosch., 29,- $€$, ISBN 978-3-8329-2465-2
}

Friedensnobelpreis 2005 für die IAEO und ihren Generaldirektor - für die Sicherung der Nuklearenergie und ihrer Anwendungen vor militärischem Missbrauch und zur Verbesserung der Sicherheit bei ihrer friedlichen Nutzung. Zum Repertoire der IAEO gehören neben den Schlaglichtern Iran, Irak und Nordkorea auch der Umweltschutz, Ernährung, die medizinische Versorgung und sauberes Trinkwasser.

Bitte bestellen Sie bei Ihrer Buchhandlung oder bei Nomos |

Telefon 07221/2104-37 | Fax -43 | www.nomos.de | sabine.horn@nomos.de

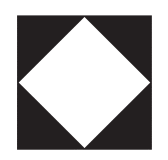

Nomos 